\title{
Dopamine D2 -141C Ins/Del and Taq1A polymorphisms, body mass index, and prediction error brain response
}

\author{
Guido K. W. Frank ${ }^{1,2}$, Megan E. Shott ${ }^{1}$, Marisa C. DeGuzman ${ }^{1,2}$ and Andrew Smolen ${ }^{3}$
}

\begin{abstract}
The prediction error model is a widely used paradigm that is conceptually based on neuronal dopamine function. However, whether dopamine receptor gene alleles contribute to human neuroimaging prediction error results is uncertain. Recent research implicated the dopamine D2 receptor in behavior response during a prediction error paradigm and we expected that polymorphisms of that receptor would contribute to prediction error brain response. In this study, healthy female participants in the early follicular phase of the menstrual cycle underwent a taste prediction error paradigm during functional magnetic resonance imaging. Participants were also genotyped for dopamine receptor polymorphisms. Our data suggest that the dopamine D2 receptor $-141 \mathrm{C}$ Ins/Del and Taq1A polymorphisms together with body mass index selectively explain putamen prediction error response. This was true using a region of interest analysis as well as for a whole-brain analysis (FWE corrected). Polymorphisms for dopamine D1 or D4 receptors, dopamine transporter, or COMT did not significantly contribute to prediction error activation. The prediction error model is a computational reward-learning paradigm that is important in psychiatric research and has been associated with dopamine. The results from this study indicate that dopamine D2 receptor polymorphisms together with body mass index are important determinants to include in research that tests prediction error response of the brain. Psychiatric disorders are frequently associated with elevated or reduced body weight. Adding BMI to genetic information in brain-imaging studies that use reward and the prediction error paradigm may be important to increase validity and reliability of results.
\end{abstract}

\section{Introduction}

Brain reward response has been associated with dopamine (DA) function ${ }^{1}$. However, identifying how DA genotype contributes to this brain activation has been challenging ${ }^{2}$. Previous studies used a "multilocus" composite DA genotype approach, where an additive score was calculated based on a presumed DA signal-enhancing vs. -reducing alleles ${ }^{2,3}$. This strategy requires knowledge of the biochemical significance of each allele on DA

\footnotetext{
Correspondence: Guido K.W Frank (Guido.Frank@ucdenver.edu) 'Department of Psychiatry, University of Colorado School of Medicine, University of Colorado Anschutz Medical Campus, Aurora, CO, USA

${ }^{2}$ Neuroscience Program, University of Colorado Denver, Anschutz Medical Campus, Aurora, CO, USA

Full list of author information is available at the end of the article
}

signaling, though, and it has been uncertain if the biological effects of the polymorphisms are additive ${ }^{2}$.

Those referenced previous studies had applied brainimaging tasks for receipt or anticipated receipt of taste or monetary reward stimuli, or a game that involved guessing numbers to win money ${ }^{2,3}$. However, reward processing involves a complex brain circuitry and various neurotransmitters, and those tasks were not necessarily anchored in a model for DA-related brain response ${ }^{4}$. An approach that has been associated with neuronal DA response is reinforcement learning, especially in the context of unexpected receipt or omission of reward stimuli ${ }^{1}$. Midbrain dopaminergic neurons exhibit a phasic burst when receiving unexpected reward ("positive prediction error"), and will shift the signal to the onset of a

\section{(c) The Author(s) 2018}

(c) Open Access This article is licensed under a Creative Commons Attribution 4.0 International License, which permits use, sharing, adaptation, distribution and reproduction cc) in any medium or format, as long as you give appropriate credit to the original author(s) and the source, provide a link to the Creative Commons license, and indicate if changes were made. The images or other third party material in this article are included in the article's Creative Commons license, unless indicated otherwise in a credit line to the material. If material is not included in the article's Creative Commons license and your intended use is not permitted by statutory regulation or exceeds the permitted use, you will need to obtain permission directly from the copyright holder. To view a copy of this license, visit http://creativecommons.org/licenses/by/4.0/. 
conditioned stimulus, which they have learned will predict reward receipt ${ }^{1}$. A negative prediction error (PE) (dip in DA neuron activity) is evoked when the predicted stimulus association is violated (unexpected reward omission, negative PE). In this model, the so-called temporal difference algorithm, a PE can be calculated based on expectation and reward outcome, which directly relates to changes in phasic or tonic DA neuron activity ${ }^{1,5,6}$. Human brain-imaging studies have shown that especially the ventral striatum (caudate, putamen) and midbrain are responsive to or code the $\mathrm{PE}^{6}$.

The PE model is part of the National Institute of Mental Health (NIMH) research domain criteria initiative (RDoC, positive valence, approach motivation) and has therefore become highly relevant for psychiatric research ${ }^{7,8}$. Understanding the involvement of specific neurotransmitter receptors in this model is of critical importance to identify specific pharmacological targets when studying disorders associated with altered DA and reward function. Although DA-D1 and DA-D2 receptors have been associated with $\mathrm{PE}$ response, the specific receptor alleles involved have been elusive, and this distinction of function has also been questioned ${ }^{9,10}$. Specifically, those reports hypothesize that phasic DA signals encode unexpected rewards and are associated with behavioral activation through D1 receptors that have low DA affinity. On the other hand, avoidance learning and behavior inhibition are mediated through the high DA affinity D2 receptors. However, such a dichotomous view may not be correct, and those receptor systems may be working more in concert than antagonistically ${ }^{9,10}$. Recent research has implicated in particular the DA-D2 receptor (DA-D2R) and one of its polymorphisms, the Taq1A allele, in a behavioral PE reinforcement learning task ${ }^{11}$. However, we are not aware of studies that have investigated the effects of DA gene polymorphisms on human PE brain response.

In this study, we investigated the role of DA-D2R alleles in driving PE response in the human brain using functional magnetic resonance brain imaging (fMRI). Eisenegger et al. $^{11}$ indicated that the DA-D2R would be involved in PE response. But the direction in which allele frequency would drive brain activation has been elusive. We therefore decided to use linear regression modeling to test those effects. Previous data comparing individuals with anorexia nervosa and obesity suggested that body mass index (BMI) could also contribute to PE response ${ }^{12}$. Starvation is associated with certain adaptations, which drive food intake ${ }^{13-15}$, including changes in DA release and receptor expression ${ }^{15}$. Similarly, overeating and associated weight gain cause changes in the DA system, and specifically in DA-D2R expression ${ }^{16}$. Those described changes occur on a continuum between under-, normaland overweight individuals ${ }^{12}$, and we therefore hypothesized that BMI would explain at least part of the variance of this response in healthy controls in addition to DA-D2 genotype. For completeness, we also explored whether we would find specific contribution of DA-D1 receptor (DAD1R) alleles to PE response, as well as polymorphisms for the catechol-o-methyltransferase (COMT), DA transporter (DAT), or the DA D4 receptor (DA-D4R), which had been included in previous studies ${ }^{2,3}$.

\section{Subjects and methods \\ Study participants}

Thirty-three healthy Caucasian females were included in the study, which was approved by the Colorado Multiple Institutional Review Board. All study participants signed informed consent. Participants ranged in age from 16 to 43 years (mean $24.3 \pm 7.3$ ) and were of normal BMI, ranging from 18.1 to 24.2 (M 21.6 \pm 1.4 ), based on the Centers for Disease and Prevention definition. Study subjects did not have a history of major medical illness and were free from DSM-5 psychiatric diagnoses as determined by a structured clinical interview conducted by a doctoral level interviewer ${ }^{17}$. All women were studied during the early phase of the follicular cycle ${ }^{18}$. This study included novel analyses and we did not have prior data to base the adequate sample size on. However, previous brain-imaging studies with 20-30 individuals per group provided significant correlational results and we expected that over 30 subjects per group would be adequate ${ }^{19}$.

\section{Genotyping}

Participants were asked to provide saliva, from which epithelial cells were collected, using a commercial product, Oragene (DNAgenotek, Kanata, Ontario, Canada). DNA was extracted from the samples using standard salting-out and solvent precipitation methods, yielding an average of $45 \mu \mathrm{g}$ of DNA. Methods for genotyping the DAT and DRD4 polymorphisms are detailed by Haberstick et al. ${ }^{20}$

Genotyping of the DA-D2R (ANKK1) Taq1A (rs1800497) and COMT Val158Met (rs4680) SNPs using Taqman are detailed in Haberstick and Smolen ${ }^{21}$.

The Taqman assay for the DRD2 -141C Ins/Del (rs1799732) utilized primers and probes from Gemignani et $a l . .^{22}$ forward primer: 5'- AAACAAGGGATGGCGGAATC-3'; reverse primer: $5^{\prime}$ CACCAAAGGAGCTGTACCTC-3'; del probe: $5^{\prime}$-VICCAACCCCTCCTACCCGTTCAGGC-MGB-3'; Ins probe: 5'-FAM-CCCTCCTACCCGTTCCAGGCMGB-3'. Taqman assays for three SNPs in DRD1, rs686 (C__1011786_10), rs4532 (C__1011777_10), and rs5326 (C_11157157_10) were purchased from Thermo Fisher Scientific. All SNP assays were performed according to manufacturer's protocols using TaqMan Genotyping Master Mix in an ABI 7000 real-time PCR system in a 
total volume of $15 \mu \mathrm{l}$ containing $2 \mu \mathrm{l}$ of genomic DNA ( $\leq 20 \mathrm{ng}$ ).

\section{Brain-imaging procedures}

On the study day, participants arrived between 7.00 and 8.00 AM after an overnight fast, and received a standardized breakfast with the instruction to eat until comfortably full. fMRI was performed between 8.00 and 9.00 AM. Brain images were acquired on a GE Sigma 3T scanner. T2* weighted echo-planar imaging for bloodoxygen dependent (BOLD) functional activity was performed, voxel size $3.4 \times 3.4 \times 2.6 \mathrm{~mm}$, TR $2100 \mathrm{~ms}$, TE $30 \mathrm{~ms}$, angle $70^{\circ}, 30$ slices, interleaved acquisition, and $2.6 \mathrm{~mm}$ slice thickness with $1.4 \mathrm{~mm}$ gap. We also acquired structural images (T1, SPGR field of view $22 \mathrm{~cm}$, flip angle $10^{\circ}$, slice thickness $1.2 \mathrm{~mm}$, scan matrix $256 \times 256$, TR 10 , TE 3, voxel size $1.2 \mathrm{~mm}^{3}$ ) for analysis of brain anatomy.

\section{Classical conditioning task}

We adapted the design used by O'Doherty et al. ${ }^{5}$ Individuals received three taste stimuli as unconditioned stimulus (US) during fMRI imaging: $1 \mathrm{M}$ Sucrose solution (100 trials), No solution (100 trials), or artificial saliva (80 trials). Individuals learned to associate each taste stimulus with a unique paired visual conditioned stimulus (CS), a geometric shape, which was only probabilistically associated with its corresponding US: the CS shape for No solution was followed in $20 \%$ of the trials by sucrose (unexpected sucrose receipt, positive PE condition), and the CS shape for Sucrose was followed in $20 \%$ of trials by No solution (unexpected sucrose omission, negative PE condition). Each visual cue (CS) was presented for $2 \mathrm{~s}$. With the disappearance of the visual cue, simultaneously the taste stimulus (US) was delivered, and a black fixation cross appeared on white background. The taste fluid delivery occurred over $1 \mathrm{~s}$. Inter-trial interval was fixed at $6 \mathrm{~s}$. Subjects were instructed to swish their tongue once, look at the fixation cross, and await the next trial. For each subject, the first 10 trials were fixed CS shape for sucrose followed by the delivery of US sucrose to establish an initial stable association between the CS sucrose shape and US sucrose taste ${ }^{5}$. All other trials were fully randomized without predetermined order. The taste stimuli were applied using a customized programmable syringe pump (J-Kem Scientific, St. Louis, MO) controlled by EPrime Software (Psychological Software Tools, Pittsburgh, PA), and individual taste applications were triggered by the MRI's scanner's radiofrequency pulse ${ }^{23,24}$. Task duration was $28 \mathrm{~min}$.

\section{Brain-imaging analysis}

Brain-imaging data were preprocessed and analyzed using SPM12 software (http://www.fil.ion.ucl.ac.uk/spm/ software/spm12/). Data from each subject were realigned to the first volume, normalized to the Montreal Neurological Institute template, and smoothed with a 6-mm FWHM Gaussian kernel. Each image sequence was manually inspected, and subjects with artifacts or movement $>3$ voxel size were excluded from the analysis. Data were preprocessed with slice time correction and motion parameters were applied as regressors in the first-level analysis.

We extracted mean parameter estimates across all voxels within predefined anatomical regions of interest (http://marsbar.sourceforge.net/) to avoid problems from small volume corrected peak voxel statistics or violation of normal distribution. We explored standard a priori bilateral $^{25}$ reward circuitry regions of interest (Automated Anatomical Labeling Atlas $\left.{ }^{26}\right)$ : bilateral caudate head, putamen, substantia nigra, and nucleus accumbens.

\section{Computational model analysis}

To test temporal difference model-related brain response, we modeled each participant's individual PE signal based on trial sequence ${ }^{5,27}$. The predicted value $(\hat{V})$ at any time $(t)$ within a trial is calculated as a linear product of weights $\left(w_{i}\right)$ and the presence of the CS stimulus at time $t$, coded in a stimulus representation vector $x_{i}(t)$ where each stimulus $x_{i}$ is represented separately at each moment in time ${ }^{5}$ :

$$
\hat{V}(t)=\sum_{i} w_{i} x_{i}(t)
$$

The predicted stimulus value at each time point $t$ in the trial is updated by comparing the predicted value at time $t$ +1 to that actually observed at time $t$, leading to the PE $\delta$ $(t)$ :

$$
\delta(t)=r(t)+\gamma \hat{V}(t+1)-\hat{V}(t),
$$

where $r(t)$ is the reward at time $t$. The parameter $\gamma$ is a discount factor, which determines the extent to which rewards arriving sooner are more important than rewards that arrive later during the task, with $\gamma=0.99^{5}$. The weights $w_{i}$ relate to how likely a particular US follows the associated CS and are updated on each trial according to the correlation between PE and the stimulus representation:

$$
\Delta w_{i}=\alpha \sum_{t} x_{i}(t) \delta(t)
$$

where $\alpha$ is a learning rate. Among various learning rates $(0.2,0.5,0.7)$, a slow $\alpha=0.7$ was the best fit for study groups ${ }^{5}$. The initial reward values were 1 for Sucrose and 0 for No solution. The PE calculated for each trial was modeled as an absolute (reflecting response strength) without separating positive or negative PE trials. This trial-to-trial calculated PE was then regressed with the parameter estimates derived from brain activation across 
all trials within each subject. Parameter estimates were then extracted for further analysis.

\section{Statistical analysis}

All analyses were conducted using the statistics package SPSS 24 (IBM, Inc.).

Table 1 Multilinear regression results for bilateral putamen; $\beta$-values are standardized and associated $p$ values derived after bootstrapping

\begin{tabular}{lllll}
\hline & $\boldsymbol{\beta}$ & $\boldsymbol{p}$ & $\begin{array}{l}\text { Collinearity } \\
\text { tolerance }\end{array}$ & ANOVA \\
\hline Right putamen & & & & \\
BMl & -0.556 & 0.001 & 0.861 & $F=7.407$, \\
DRD2 -141 Ins/Del & 0.396 & 0.018 & 0.887 & $p<0.001$ \\
DRD2 Taq1A & -0.461 & 0.001 & 0.96 & \\
Left putamen & & & & \\
BMl & -0.457 & 0.002 & 0.861 & $F=6.592$, \\
DRD2 -141 Ins/Del & 0.434 & 0.035 & 0.887 & $p<0.002$ \\
DRD2 Taq1A & -0.477 & 0.001 & 0.96 & \\
\hline
\end{tabular}

We created a composite score for each participant based on each DA receptor's genotype and alleles per participant. For each region of interest, the mean value for the PE model regression parameter estimates was extracted and included in a multiple linear regression analysis. Each region of interest-extracted brain parameter estimate was considered the dependent variable. BMI as well as the DA receptor genotypes were included as independent variables. The primary hypothesis was based on DA-D2R polymorphisms driving PE response together with BMI and those variables were grouped together (PE (dependent)-BMI, DA-D2R polymorphisms (independents)). In addition, the DA-D1R polymorphisms rs686, rs4532, and rs5326 were grouped with BMI (PE (dependent)-BMI, DA-D1R polymorphisms (independents)), as were COMT, DAT, and DA-D4R (PE (dependent-BMI, COMT, DAT, DA-D4R (independents)). Linear regression was performed using the "Enter" method. This method was selected because we did not have a specific hypothesis, which of the independent variables would produce the best prediction equation. This is also the most stringent method for multiple linear regression. In addition, we performed bootstrapping. This resampling method was used to reduce bias. In the multiple regression analysis, we assessed collinearity, and a variance
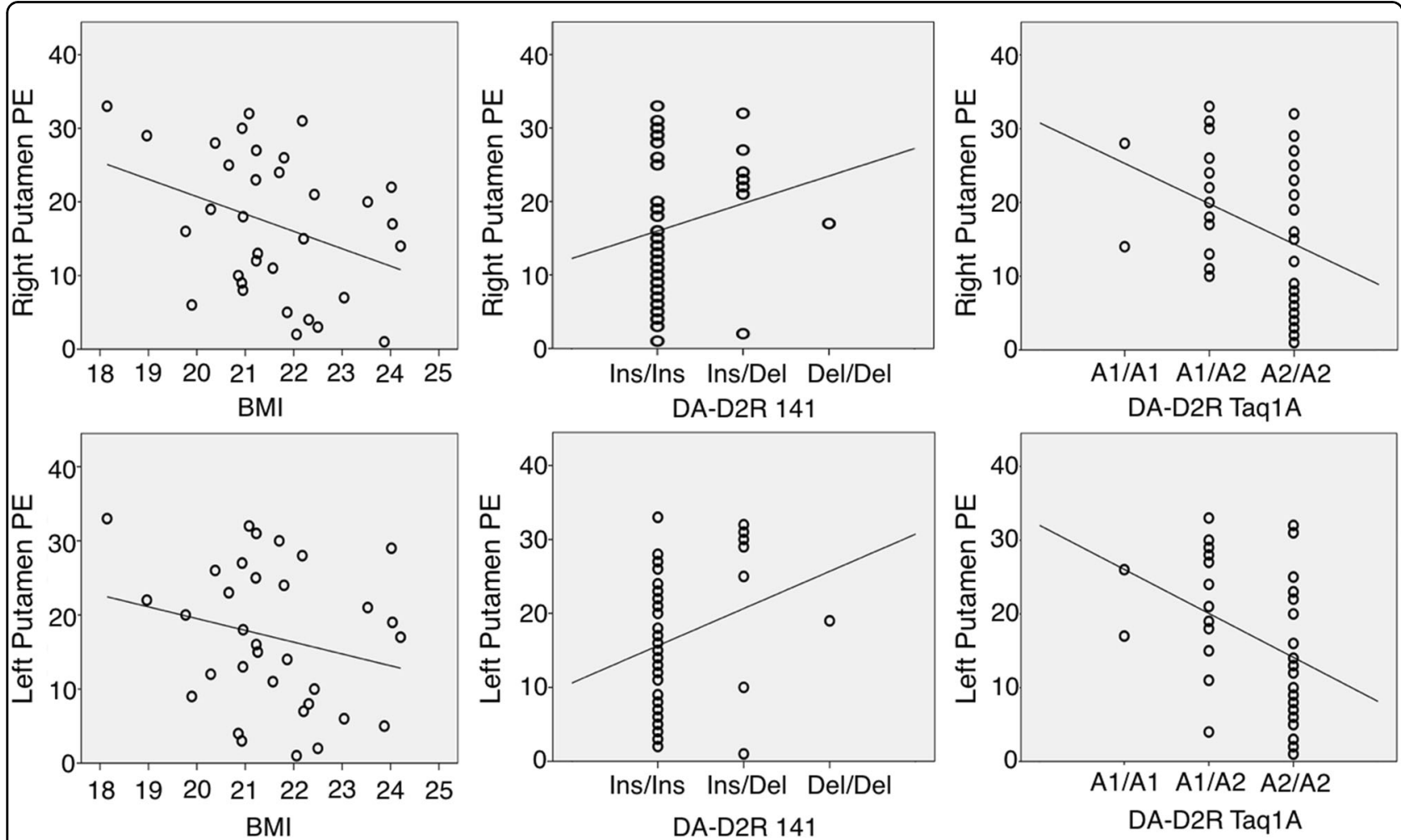

Fig. 1 Prediction error correlation plots. Individual scatter plots for body mass index (BMI), DA-D2R alleles for the 141 and Taq1A genotype and bilateral putamen prediction error (PE) values (top panel: right putamen; bottom panel: left putamen) 

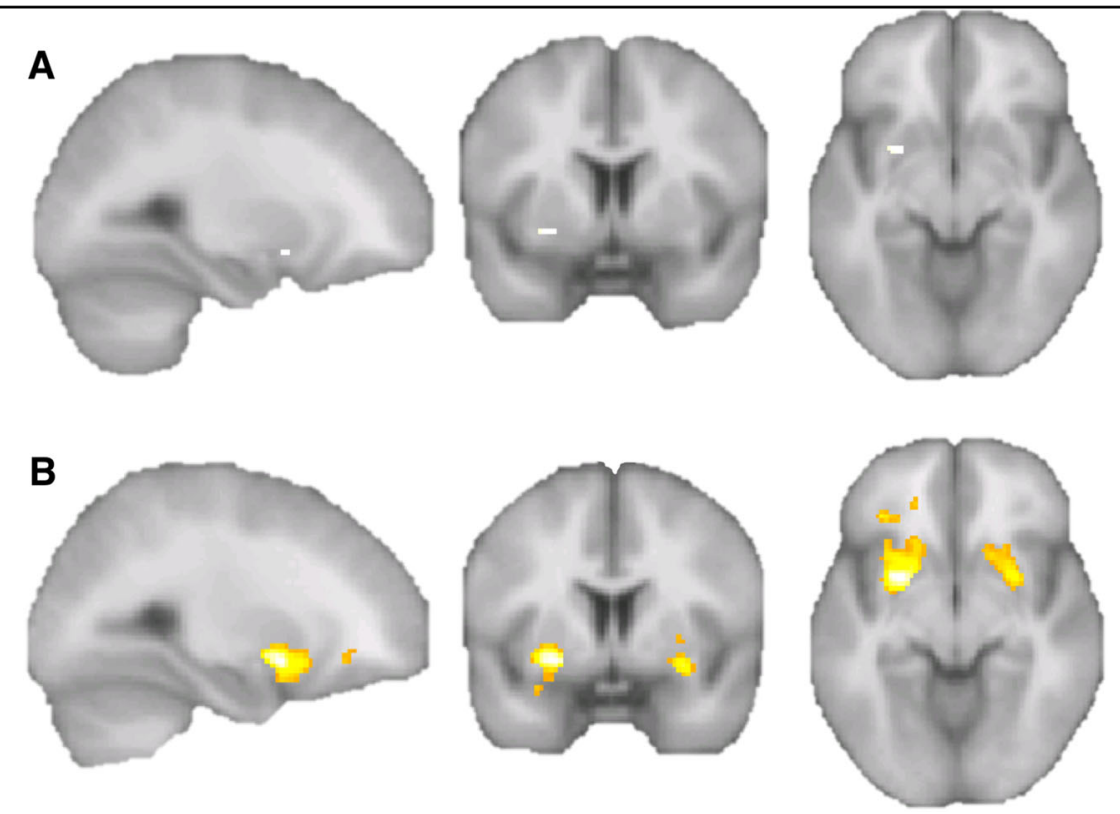

Fig. 2 Whole-brain regression between summary scores of BMI, DA-D2R 141, and Taq1A genotype score with prediction error (PE) maps; no mask was applied. a Threshold $p<0.05$ FWE corrected; one significant cluster, $x=26, y=4, z=-8$, peak $p_{\mathrm{FWE}}<0.018, k=8$, right putamen, cluster $p_{\mathrm{FWE}}<0.002$. b An additional regression at lower threshold $(p<0.001$, uncorrected) indicated that the BMl and genotype score correlated almost exclusively with ventral putamen PE activation (cluster $p_{\mathrm{FWE}}<0.001$ and $p<0.002$, right and left hemispheres, see supplemental material for full results)

inflation factor of $>5$ was considered indicating significant collinearity. Non-normally distributed data were ranktransformed before statistical analysis.

We further computed a summary score (addition) for the DA-D2R Taq1A alleles, -141C Ins/Del alleles and $\mathrm{BMI}$ and performed a whole-brain regression with $\mathrm{PE}$ regression maps. For the DA-D2R 141, the Del/Del genotype was assigned a value of 1 , the Ins/Del 2 , and Ins/Ins 3; for DA-D2R Taq1A, a value of 1 was assigned to the $\mathrm{A} 1 / \mathrm{A} 1$, a 2 to the $\mathrm{A} 1 / \mathrm{A} 2$, and a 3 to the $\mathrm{A} 2 / \mathrm{A} 2$ genotype. Those values were added to the raw BMI value and regressed with brain response. Regression maps were thresholded at $p<0.05$ peak voxel FWE corrected. In order to test whether a regression would be region-specific, we created a regression map at $p<0.001$ uncorrected.

\section{Results}

\section{Genotype data were within the Hardy-Weinberg equilibrium}

The combination of DA-D2R Taq1A, -141C Ins/Del and BMI was highly predictive of bilateral putamen PE response and survived multiple comparison correction (Bonferroni), but not for other regions (Table 1, Fig. 1). Figure 1 also shows directionality of DA-D2R allele as well as BMI effects on PE. There was no interaction between BMI and allele frequency. DA-D1R alleles or DAT, COMT, or D4 together with BMI did not significantly predict PE response. However, coefficients for DA-D1R alleles rs686, rs4532 in bilateral nucleus accumbens were significant, and after exploratory removal of the rs5326 allele and BMI, the rs686 and rs4532 alleles predicted left nucleus accumbens PE response $(F=3.933, p<0.03)$, but did not survive multiple comparison correction.

We further computed a summary score (addition, supplemental material) for the DA-D2R Taq1A alleles, $-141 \mathrm{C}$ Ins/Del alleles, and BMI, and performed a wholebrain regression with $\mathrm{PE}$ regression maps. That showed a cluster in the right putamen $(p<0.05$, FWE corrected, whole brain unmasked; Fig. 2, Supplemental Table 1), which was significant at the cluster level $(p<0.002)$. An additional exploratory analysis at lower significance threshold $(p<0.001$ uncorrected, 50 voxel cluster threshold) indicated across the whole brain almost exclusive regression with putamen brain response, although there were three additional smaller clusters in the middle and inferior prefrontal cortex. In that analysis, the bilateral putamen clusters were significant $p<0.05$ FWE corrected at the cluster level (right $p<0.001$, left $p<$ 0.002).

\section{Discussion}

This study provides empirical evidence that DA-D2R alleles contribute together with BMI to human PE response in the putamen. The DA-D2R 141 Ins/Ins and Taq1A A2/A2 alleles and higher BMI were associated 
with lower PE response. DA-D1R allelic variation might be a factor in nucleus accumbens response, but we did not find evidence that DAT, COMT, or DA-D4R contribute to this response. Previous studies using computational algorithms found that in particular the putamen responds to PE tasks ${ }^{4,5,28}$. Our results are consistent with those findings.

From these results, we can propose a model for how the combination of genotype and BMI can explain a significant amount of variance in PE response. This is novel and has not been shown before. This is important for various reasons. First, it highlights the importance of DA-D2R allele frequency and proposing a mechanism for variation of $\mathrm{PE}$ response across individuals. Second, the importance of the DA-D2R in general PE response indicates that DA-D2R active medication may be useful in modifying PE response, which could have clinical implications. Third, the results suggest that variation in $\mathrm{BMI}$ is also associated with changes in PE, which is important as it suggests a mechanism for how eating behavior can affect DA-related brain response. Our study is a direct extension of the study by Eisenegger et al. ${ }^{11}$ that emphasized the DA-D2R and the Taq1A allele in reinforcement learning in humans ${ }^{11}$. That study investigated male participants only, while our study was restricted to adult females. Sex and also the state of menstrual cycle modify brain reward response, and future studies need to investigate those differences further across sexes $^{29}$.

The NIMH RDoC project includes the PE model and this paradigm has been now applied in a host of studies including depression and psychotic disorders ${ }^{30,31}$. This makes it even more important to understand the factors that drive the PE signal, in order to properly contrast brain response between healthy individuals and those with specific psychiatric disorders. This study adds evidence to the long hypothesized genetic underpinnings, specifically DA-D2R alleles, driving PE response ${ }^{9}$. The DA-D2R adapts to food intake and can be modified by the type of diet and weight gain ${ }^{16,32}$. This has important implications not only for conditions such as obesity and eating disorders, but also for depression and schizophrenia, conditions also often associated with high or low weight ${ }^{16,33}$. It may therefore be key to assess DA receptor genotype and BMI, and take those effects into consideration when analyzing and interpreting studies that use this paradigm. Our previous data showed that extremes of under and overweight are associated with opposite PE response ${ }^{12}$. Here we studied healthy control individuals and we believe that being able to demonstrate those effects across the spectrum of normal weight suggests a strong effect of the variables studied. Nevertheless, future studies will need to study genotype and DA-D2R effects on PE response across the spectrum of psychiatric populations.

\section{Limitations}

Our study did not measure a specific behavior response. However, choice and motivation have been associated with DA-D2R and PE response ${ }^{34}$. We only studied females and a comprehensive study is needed across both sexes. The sample is modest and requires replication, and in order to mitigate this, we used bootstrap procedures. The effects of the DA-D1R alleles did not survive multiple comparisons, however in a larger sample, their effects could have been significant. The correlation results are primarily driven by the major allele homozygotes and the heterozygotes. The number of minor allele homozygote individuals is small and their true mean value for $\mathrm{PE}$ response is difficult to assess. The data from this healthy control sample do not allow any inference on how genotype and BMI interact with psychiatric illness effects. Basic research has implicated the DA-D2R in reinforcement learning response in brain regions including striatum, pallidum, nucleus accumbens core, and habenula, but human fMRI is not necessarily well positioned to make such fine distinctions ${ }^{35-38}$. The calculated PE was modeled as absolute or strength of $\mathrm{PE}$ and regressed with the brain response reflected in the parameter estimates as in previous studies ${ }^{12,19}$. This approach does not separate by positive and negative activation, but has been selected because it is uncertain whether DA receptor function can be separated for positive and negative PE response ${ }^{10}$. We included a broad age range in the analysis for study of a larger population sample. In order to test for age effects, we ran an additional exploratory analysis, which also included age in the model. However, while BMI effects remained significant for right $(p<0.001)$ and left $(p<$ $0.031)$ putamen, there were no significant effects for age for right $(p<0.495)$ - or left $(p<0.695)$-sided putamen PE response. We therefore do not believe that the age range confounded the study results.

In summary, these data suggest that DA-D2R allelic variation contributes to $\mathrm{PE}$ response in healthy women together with BMI. This suggests that studies that apply the PE model should take those variables into account.

\footnotetext{
Acknowledgements

We would like to thank all the individuals who participated in this study. G.K.W.F. had full access to all of the data in the study and takes responsibility for the integrity of the data and the accuracy of the data analysis. This work was supported by the NIMH grants MH096777 and MH103436 and a Davis Foundation Award of the Klarman Family Foundation Grants Program in Eating Disorders (all PIGKWF) and and M.C.D. was supported by NIH grant T32HD041697 (University of Colorado Neuroscience Program).
}

\section{Author details}

${ }^{1}$ Department of Psychiatry, University of Colorado School of Medicine, University of Colorado Anschutz Medical Campus, Aurora, CO, USA.

${ }^{2}$ Neuroscience Program, University of Colorado Denver, Anschutz Medical Campus, Aurora, CO, USA. ${ }^{3}$ University of Colorado Boulder, Institute for Behavioral Genetics, Boulder, CO, USA 


\section{Conflict of interest}

The authors declare that they have no conflict of interest.

\section{Publisher's note}

Springer Nature remains neutral with regard to jurisdictional claims in published maps and institutional affiliations.

Supplementary Information accompanies this paper at https://doi.org/ 10.1038/s41398-018-0147-1.

Received: 9 October 2017 Revised: 5 February 2018 Accepted: 4 April 2018 Published online: 23 May 2018

\section{References}

1. Schultz, W., Dayan, P. \& Montague, P. R. A neural substrate of prediction and reward. Science 275, 1593-1599 (1997).

2. Nikolova, Y. S., Ferrell, R. E., Manuck, S. B. \& Hariri, A. R. Multilocus genetic profile for dopamine signaling predicts ventral striatum reactivity. Neuropsychopharmacology 36, 1940-1947 (2011).

3. Stice, E., Yokum, S., Burger, K., Epstein, L. \& Smolen, A. Multilocus genetic composite reflecting dopamine signaling capacity predicts reward circuitry responsivity. J. Neurosci. 32, 10093-10100 (2012).

4. Haber, S. N. \& Knutson, B. The reward circuit: linking primate anatomy and human imaging. Neuropsychopharmacology 35, 4-26 (2010).

5. O'Doherty, J. P., Dayan, P., Friston, K., Critchley, H. \& Dolan, R. J. Temporal difference models and reward-related learning in the human brain. Neuron 38, 329-337 (2003).

6. D'Ardenne, K., McClure, S. M., Nystrom, L. E. \& Cohen, J. D. BOLD responses reflecting dopaminergic signals in the human ventral tegmental area. Science 319, 1264-1267 (2008).

7. Insel, T. et al. Research domain criteria (RDoC): toward a new classification framework for research on mental disorders. Am. J. Psychiatry 167, 748-751 (2010).

8. NIMH-RDoC-working-group (ed). Positive Valence Systems: Workshop Proceedings (National Institute of Mental Health (NIMH), Rockville, 2011) https://www. nimh.nih.gov/research-priorities/rdoc/positive-valence-systems-workshopproceedings.shtml.

9. Maia, T. V. \& Frank, M. J. From reinforcement learning models to psychiatric and neurological disorders. Nat. Neurosci. 14, 154-162 (2011).

10. Soares-Cunha, C., Coimbra, B., Sousa, N. \& Rodrigues, A. J. Reappraising striatal D1- and D2-neurons in reward and aversion. Neurosci. Biobehav. Rev. 68, 370-386 (2016).

11. Eisenegger, C. et al. Role of dopamine D2 receptors in human reinforcement learning. Neuropsychopharmacology 39, 2366-2375 (2014).

12. Frank, G. K. et al. Anorexia nervosa and obesity are associated with opposite brain reward response. Neuropsychopharmacology 37, 2031-2046 (2012).

13. Branch, S. Y. et al. Food restriction increases glutamate receptor-mediated burst firing of dopamine neurons. J. Neurosci. 33, 13861-13872 (2013).

14. Carr, K. D., Tsimberg, Y., Berman, Y. \& Yamamoto, N. Evidence of increased dopamine receptor signaling in food-restricted rats. Neuroscience 119, 1157-1167 (2003).

15. Thanos, P. K., Michaelides, M., Piyis, Y. K., Wang, G. J. \& Volkow, N. D. Food restriction markedly increases dopamine D2 receptor (D2R) in a rat model of obesity as assessed with in-vivo muPET imaging ([11C] raclopride) and in-vitro ([3H] spiperone) autoradiography. Synapse 62, 50-61 (2008).

16. Johnson, P. M. \& Kenny, P. J. Dopamine D2 receptors in addiction-like reward dysfunction and compulsive eating in obese rats. Nat. Neurosci. 13, 635-641 (2010).
17. American Psychiatric Association. Desk Reference to the Diagnostic Criteria from DSM-5 Vol. xlviii (American Psychiatric Publishing, Washington, DC, 2013).

18. Dreher, J. C. et al. Menstrual cycle phase modulates reward-related neural function in women. Proc. Natl Acad. Sci. USA 104, 2465-2470 (2007).

19. DeGuzman, M., Shott, M. E., Yang, T. T., Riederer, J. \& Frank, G. K. W. Association of elevated reward prediction error response with weight gain in adolescent anorexia nervosa. Am. J. Psychiatry 174, 557-565 (2017).

20. Haberstick, B. C. et al. Simple sequence repeats in the national longitudinal study of adolescent health: an ethnically diverse resource for genetic analysis of health and behavior. Behav. Genet. 44, 487-497 (2014).

21. Haberstick, B. C. \& Smolen, A. Genotyping of three single nucleotide polymorphisms following whole genome preamplification of DNA collected from buccal cells. Behav. Genet. 34, 541-547 (2004).

22. Gemignani, F. et al. Polymorphisms of the dopamine receptor gene DRD2 and colorectal cancer risk. Cancer Epidemiol. Biomarkers Prev. 14, 1633-1638 (2005).

23. Frank, G. K., Reynolds, J. R., Shott, M. E. \& O'Reilly, R. C. Altered temporal difference learning in bulimia nervosa. Biol. Psychiatry 70, 728-735 (2011).

24. Frank, G. et al. The evaluation of brain activity in response to taste stimuli-a pilot study and method for central taste activation as assessed by eventrelated fMRI. J. Neurosci. Methods 131, 99-105 (2003).

25. Shott, M. E., Pryor, T. L., Yang, T. T. \& Frank, G. K. Greater insula white matter fiber connectivity in women recovered from anorexia nervosa. Neuropsychopharmacology 41, 498-507 (2016).

26. Tzourio-Mazoyer, N. et al. Automated anatomical labeling of activations in SPM using a macroscopic anatomical parcellation of the MNI MRI singlesubject brain. Neuroimage 15, 273-289 (2002).

27. Schultz, W. Predictive reward signal of dopamine neurons. J. Neurophysiol. $\mathbf{8 0}$ 1-27 (1998).

28. McClure, S. M., Berns, G. S. \& Montague, P. R. Temporal prediction errors in a passive learning task activate human striatum. Neuron 38, 339-346 (2003).

29. Alarcon, G., Cservenka, A. \& Nagel, B. J. Adolescent neural response to reward is related to participant sex and task motivation. Brain. Cogn. 111, 51-62 (2017).

30. Chen, C., Takahashi, T., Nakagawa, S., Inoue, T. \& Kusumi, I. Reinforcement learning in depression: a review of computational research. Neurosci. Biobehav. Rev. 55, 247-267 (2015).

31. Deserno, L., Schlagenhauf, F. \& Heinz, A. Striatal dopamine, reward, and decision making in schizophrenia. Dialogues Clin. Neurosci. 18, 77-89 (2016).

32. Sharpe, M. J., Clemens, K. J., Morris, M. J. \& Westbrook, R. F. Daily exposure to sucrose impairs subsequent learning about food cues: a role for alterations in ghrelin signaling and dopamine D2 receptors. Neuropsychopharmacology 41, 1357-1365 (2016).

33. van de Giessen, E., la Fleur, S. E., de Bruin, K., van den Brink, W. \& Booij, J. Free-choice and no-choice high-fat diets affect striatal dopamine D2/3 receptor availability, caloric intake, and adiposity. Obesity 20, 1738-1740 (2012).

34. Kelley, A. E. \& Berridge, K. C. The neuroscience of natural rewards: relevance to addictive drugs. J. Neurosci. 22, 3306-3311 (2002).

35. Nakajima, S. et al. Dopamine D2/3 receptor occupancy following dose reduction is predictable with minimal plasma antipsychotic concentrations: an open-label clinical trial. Schizophr. Bull. 42, 212-219 (2016).

36. Li, S. S. \& McNally, G. P. A role of nucleus accumbens dopamine receptors in the nucleus accumbens core, but not shell, in fear prediction error. Behav. Neurosci. 129, 450-456 (2015).

37. Jocham, G., Klein, T. A. \& Ullsperger, M. Differential modulation of reinforcement learning by D2 dopamine and NMDA glutamate receptor antagonism. J. Neurosci. 34, 13151-13162 (2014).

38. Wiecki, T. V., Riedinger, K, von Ameln-Mayerhofer, A., Schmidt, W. J. \& Frank, M. J. A neurocomputational account of catalepsy sensitization induced by D2 receptor blockade in rats: context dependency, extinction, and renewal. Psychopharmacology 204, 265-277 (2009). 